\title{
La voix du gardien du lion, ou les significations multiples des animaux de la ménagerie du Muséum d'Histoire Naturelle
}

The voice of the lion keeper, or the multiple meanings of the animals of the menagerie of the Muséum d'Histoire Naturelle

\section{Richard W. Burkhardt}

Traducteur : Lucie Perrier

\section{CpenEdition} Journals

\section{Édition électronique}

URL : https://journals.openedition.org/ahrf/13287

DOI : $10.4000 /$ ahrf. 13287

ISSN : 1952-403X

Éditeur :

Armand Colin, Société des études robespierristes

Édition imprimée

Date de publication : 15 octobre 2014

Pagination : 145-173

ISBN : 978-2-20-092927-5

ISSN : 0003-4436

Référence électronique

Richard W. Burkhardt, "La voix du gardien du lion, ou les significations multiples des animaux de la ménagerie du Muséum d'Histoire Naturelle», Annales historiques de la Révolution française [En ligne], 377 | juillet-septembre 2014, mis en ligne le 15 septembre 2017, consulté le 01 juillet 2021. URL : http://journals.openedition.org/ahrf/13287 ; DOI : https://doi.org/10.4000/ahrf.13287 


\title{
LA VOIX DU GARDIEN DU LION, OU LES SIGNIFICATIONS MULTIPLES DES ANIMAUX DE LA MÉNAGERIE DU MUSÉUM D'HISTOIRE NATURELLE
}

Richard W. BURKHARDT, JR.

\begin{abstract}
Pendant la Révolution, on considéra le Muséum national d'Histoire Naturelle comme un lieu où l'exposition d'images convenables de la Nature contribuerait à élever la moralité publique. À la nouvelle ménagerie du Muséum, on prétendait que les citoyens apprendraient à se mieux comporter vis-à-vis de leurs semblables s'ils constataient que la nature animale s'améliorait grâce à un traitement bienveillant, à la sociabilité, et à la jouissance d'une liberté relative. II y avait cependant un gouffre entre la théorie et la pratique. Il était compliqué de mettre en œuvre les scénarios désirés, et difficile de maîtriser les conclusions que le public tirait de ce qu'il voyait. Ici nous mettons l'accent sur les lions de la ménagerie, sur les commentaires variés, parfois contradictoires, dont ils firent l'objet et les entreprises humaines qui en découlèrent. Alors que Lacepède suggérait qu'il serait préférable qu'un jardin zoologique moderne n'ait pas de lions, le public souhaitait voir des lions, le gardien des animaux féroces voulait en montrer, et le gouvernement les exhibait en témoignage de la grandeur de la France et de son rayonnement dans le monde.
\end{abstract}

Mots-clés : ménagerie, lions, animaux féroces, Muséum national d'histoire naturelle, Lacepède, Bernardin de Saint-Pierre, Frédéric Cuvier, Félix Cassal. 
À l'automne 1792, Bernardin de Saint-Pierre ${ }^{1}$, en sa qualité de nouvel Intendant du Jardin des Plantes, se rendit de Paris à Versailles avec deux de ses collègues pour voir les animaux qui restaient de l'ancienne ménagerie royale. Accompagnés du régisseur général des domaines de Versailles et du concierge de la ménagerie, les visiteurs observèrent les derniers animaux du jardin zoologique : un rhinocéros, un quagga, un bubale, un pigeon à crête bleue, et un lion qui avait un chien pour compagnon. Comme on l'attendait d'un auteur connu pour l'attention qu'il portait aux relations harmonieuses entre l'homme et la nature, Bernardin s'intéressa à la manière dont chacun des animaux réagissait à l'arrivée des visiteurs. L'oiseau courait çà et là au fond de sa cage «dans une agitation perpétuelle » mais le rhinocéros, le bubale et le couagga s'approchaient afin de se faire caresser à travers les barreaux de leurs enclos. Le chien et le lion s'avançaient également, le chien remuant la queue en signe de bienvenue, alors que le lion «se promenait gravement le long de ses barreaux, contre lesquels il frottait sa tête énorme ». Dans ce petit groupe, le lion et le chien furent les animaux qui impressionnèrent le plus Bernardin :

« Jamais je n'avais vu tant de générosité dans un lion, et tant d'amabilité dans un chien. Celui-ci sembla deviner que sa familiarité avec le roi des animaux était le principal objet de notre curiosité. Cherchant à nous complaire dans sa captivité, dès que nous lui eûmes adressé quelques paroles d'affection, il se jeta d'un air gai sur la crinière du lion, et lui mordit en jouant les oreilles. Le lion, se prêtant à ses jeux, baissa la tête et fit entendre de sourds rugissements $»^{2}$.

Pour Bernardin, l'amitié entre les deux animaux lui semblait être l'« un des plus touchants spectacles que la nature puisse offrir aux spéculations d'un philosophe $»^{3}$. L'attitude du lion était « une preuve de ce que peut l'influence de la société sur le caractère le plus sauvage ; il est beaucoup plus gai qu'un lion solitaire ${ }^{4}$. Bernardin considéra ceci comme comparable à ce que le réformateur de prisons britannique John Howard avait conclu à propos de la réhabilitation morale des prisonniers : afin

(1) Sur Bernardin, voir Roselyne REY, «L'animalité dans l'œuvre de Bernardin de SaintPierre, » Revue de synthèse, 113, 1992, p. 311-331.

(2) Jacques BERNARDIN DE SAINT-PIERRE, Mémoire sur la nécessité de joindre une ménagerie au jardin national des plantes de Paris, Paris, Didot, 1792, p. 10-11.

(3) Ibidem, p. 9.

(4) Ibid., p. 18. 
de corriger les détenus de leurs mauvaises habitudes, il ne fallait pas les abandonner à la solitude 5 .

Les observations de Bernardin parurent en décembre 1792 dans un mémoire où il proposait qu'une ménagerie soit installée au Jardin des Plantes. Ce dont cette institution avait le plus besoin, insistait-il, était la présence d'animaux vivants, c'est-à-dire d'animaux « qui sentent, qui aiment, qui connaissent $»^{6}$. Pour éclairer les exigences et la nature des hommes, disait-il, il était nécessaire d'être à même d'étudier les « goûts, les instincts et les passions » des animaux, pas uniquement leur anatomie. Les descriptions d'animaux vivants furent ce qui « a rendu Buffon si intéressant, non seulement aux savants, mais à tous les hommes $\gg^{7}$. En outre, pour faire progresser la moralité publique, il serait largement préférable de voir des animaux vivants traités avec bienveillance plutôt que de regarder des spécimens morts dont la vie leur avait été brutalement arrachée «par nos naturalistes meurtriers! ». La férocité des hommes les uns vis-à-vis des autres, expliquait Bernardin, " commence souvent son apprentissage par celle qu'il voit exercer sur les animaux $»^{8}$.

Le point de vue de Bernardin sur les bénéfices pour la société qui découleraient d'une ménagerie bien gérée se rapporte directement à ce que nous avons, ailleurs, décrit comme une entreprise de « civilisation des spécimens et des citoyens $»{ }^{9}$. Ceci était, en effet, ce que le nouveau Muséum d'histoire naturelle, qui succédait à l'ancien Jardin du Roi, s'engageait à accomplir. De fait, estimer qu'elle convenait bien à cette tâche fut ce qui conféra à cette institution une position particulière au cours de la Révolution à une époque où d'autres anciens établissements royaux étaient définitivement fermés. La conservation et l'augmentation des collections d'histoire naturelle pour l'instruction publique aussi bien que pour le progrès de la science ; la poursuite de programmes pratiques d'acclimatation, de domestication, de reproduction, de multiplication et de distribution de plantes et animaux utiles; la présentation méthodique d'images de la Nature qui aidaient les citoyens à comprendre leur place en son sein et dans la société - ces activités donnaient au Muséum sa légitimité sociale. Les

(5) Ibid., p. 17.

(6) Ibid., p. 3.

(7) Ibid., p. 4.

(8) Ibid., p. 40.

(9) Richard W BurKHARDT, Jr., « Civilizing Specimens and Citizens at the Muséum d'Histoire Naturelle, 1793-1838 », dans Sue Ann PRINCE (dir.) Of Elephants and Roses : French Natural History, 1790-1830, Memoirs of the American Philosophical Society, 267, 2013, p. 1-15. Voir également Richard W. BuRKHARDT, «The Leopard in the Garden : Life in Close Quarters at the Muséum d'Histoire Naturelle », Isis, 98, 2007, p. 675-694. 
naturalistes tirèrent profit du fait qu'ils pouvaient prétendre que leur travail n'avait pas un caractère élitiste. Leur cause fut en outre servie parce que les jardins botaniques étaient considérés comme des lieux de transformation matérielle et morale ${ }^{10}$.

Cela dit, cependant, l'endroit où des animaux tels que les léopards, ours polaires et mandrills se situaient dans ce tableau n'était pas clairement défini. La question s'imposa aux professeurs/administrateurs du Muséum en novembre 1793, lorsque, à la suite d'un décret de la Commune de Paris, un certain nombre de ces animaux furent livrés au Muséum après qu'ils avaient été confisqués aux forains qui les possédaient et les avaient exhibés dans les rues et sur les places de Paris. Léopards et ours polaires n'étaient pas le genre de bêtes qui apparaissaient sur les listes des naturalistes aux fins d'être acclimatées et multipliées pour le bien du public. Il n'était pas davantage aisé d'imaginer comment le spectacle de tels animaux confinés dans d'étroites cages servirait à élever la moralité des foules. En réalité, l'idée même d'un jardin zoologique était difficilement compatible avec les idéaux révolutionnaires parce que la collection d'animaux la plus connue en France était l'ancienne ménagerie royale à Versailles, institution remarquable de par son caractère ostentatoire et dispendieux. Lorsque, en 1792, Bernardin de Saint-Pierre conseilla de déménager les derniers animaux de Versailles vers le Jardin des Plantes, il insista sur le fait que la ménagerie qu'il souhaitait créer en ce lieu ne ressemblerait en rien à celle de Versailles qui n'avait été « pour la nation qu'un objet inutile de luxe et de dépense $\gg^{11}$.

L'histoire des débuts de la ménagerie du Muséum d'histoire naturelle a été assez bien documentée ; nous ne tenterons pas de passer en revue ici tous les hauts et les bas qu'elle a connus au cours de la première décennie de son existence ${ }^{12}$. Notre but est plutôt d'examiner les significations multiples

(10) Voir particulièrement Emma C. SPARY, Utopia's Garden : French Natural History from Old Regime to Revolution, Chicago, U Chicago Press, 2000.

(11) Jacques BernARdin DE SAINT-PIERRE, Mémoire... op. cit., p. 5. Les spécialistes ont également remarqué la connotation négative de «ménagerie » dans l'Encyclopédie. Voir, par exemple, Louise ROBBINS, Elephant slaves and pampered parrots : exotic animals in eighteenth-century Paris, Baltimore, Johns Hopkins UP, 2002, p. 60-61; et Éric BARATAY, « La promotion de l'animal sensible. Une révolution dans la Révolution », Revue Historique, 314 (2012), p. 131-153.

(12) Voir Ernest Théodore HAMY, «Les anciennes ménageries royales et la ménagerie nationale fondée le 14 brumaire an II », Nouvelles archives du Muséum, $4^{\mathrm{e}}$ série, t. V, 1893, p. 1-22 ; Gustave LOISEL, « Histoire de la ménagerie du Muséum », Revue Scientifique, 1911, p. 262-277, 301304 ; Yves LAISsus et Jean-Jacques PETTER, Les Animaux du Muséum, 1793-1993, Paris, Imprimerie Nationale, 1993 ; Richard W. BURKHARDT, Jr., « La ménagerie et la vie du Muséum », dans Claude BLANCKAERT et al. (dir.), Le Muséum au premier siècle de son histoire, Paris, Muséum national d'histoire naturelle, Archives, 1997, p. 481-508 ; Louise RoBBINS, Elephant slaves, op. cit. 
que revêtaient les animaux qui s'y trouvaient. Elles ne furent pas seulement l'expression des théoriciens sur ce que la ménagerie pouvait être, tels Bernardin de Saint-Pierre ou Lacepède, mais d'autres également. Des personnalités du gouvernement, des individus pris dans le public, des propriétaires d'animaux expropriés qui devinrent leurs gardiens au Muséum, des surveillants du jardin zoologique, des professeurs/administrateurs au Muséum, des auteurs de guides sur cette institution ou sur la ménagerie, des journalistes, artistes, chefs d'états étrangers, et d'autres : chacun de ces différents acteurs avait ses propres intérêts, ses priorités et points de vue personnels lorsqu'il s'agissait des animaux de la ménagerie du Muséum d'histoire naturelle.

Le nombre de cas potentiels à examiner devient encore plus décourageant si l'on considère la quantité de races animales distinctes que comportait cette ménagerie. À la fin de l'année 1800, elle abritait au moins vingt espèces de mammifères et presque le double d'espèces d'oiseaux. Parmi les mammifères se trouvaient des lions, tigres, ours, chameaux, éléphants, cerfs, moutons, chèvres, un zébu, un mandrill, et d'autres encore. La signification pour le visiteur voyant un « animal féroce » enfermé dans une cage allait très vraisemblablement être différente de celle inspirée par un animal «paisible » ou « innocent», libre de se déplacer dans son parc et qui, à la vue d'un être humain, venait en toute confiance vers les limites de son enclos pour se faire caresser ou nourrir. En outre, les différences supposées ou manifestes dans le comportement d'espèces assez semblables, comme le lion et le tigre, pouvaient influencer la façon dont ces animaux étaient perçus.

Comme si la multiplication du nombre des divers acteurs humains par celui des différents animaux n'était pas suffisante, notre sujet se complique encore plus du fait de changements dans le temps. Les possessions de la ménagerie varièrent à la suite de nouvelles acquisitions d'une part, et de pertes (surtout causées par la mort) d'autre part. De plus, les variations dans le contexte politique et la situation économique eurent une incidence sur la manière dont on comprenait les animaux, tout comme les modifications dans leurs conditions d'existence. Au cours de la période en question, les aménagements de fortune qui avaient caractérisé les tout débuts de la ménagerie commencèrent à être remplacés par des éléments de "la ménagerie nouvelle ». Dès l'été 1803 , les journalistes faisaient l'éloge de la «métamorphose étonnante » de plusieurs arpents de terre qui avaient récemment été rattachés au Muséum. Ce qui, auparavant, avait été un terrain marécageux occupé par des dépôts de bois et des jardins maraîchers avait été transformé en un lieu charmant où serpentaient des chemins que 
les promeneurs parcouraient à pied, longeant des parcs pittoresques où se trouvaient des animaux exotiques. Ceux qui fréquentaient cet endroit spécial s'étaient mis à l'appeler la Vallée suisse ${ }^{13}$.

Étant donné les différents observateurs, la diversité des espèces d'animaux, les changements de contexte historique et les modifications des équipements matériels dans lesquels ces animaux étaient présentés, comment devrions-nous tenter de comprendre ce que les hôtes de la ménagerie étaient censés signifier? Afin d'essayer d'apporter quelque ordre dans une situation chaotique, nous avons décidé de limiter notre sujet. Nous laisserons de côté les « animaux paisibles », et porterons notre regard sur les « animaux féroces », parmi lesquels les lions feront l'objet d'une attention particulière. Ces derniers jouèrent un rôle primordial lorsqu'il fut question de réfléchir sur les relations aux animaux, ou ce que la ménagerie elle-même signifiait. S'occuper en particulier des lions est utile pour étudier la question des modifications de l'agencement matériel du jardin zoologique car, à cette époque, - et en dépit des intentions contraires du Muséum - les « animaux féroces » restèrent dans le même bâtiment délabré, une serre aménagée près de la Seine. Sur cette construction, au printemps 1794, le Muséum accrocha un panneau d'excuse : «ménagerie provisoire ».

Avant d'aborder les lions, disons encore quelques mots sur « la civilisation des spécimens et des citoyens ». L'importance à accorder à la maîtrise ou la « civilisation » des animaux du jardin zoologique devrait être assez évidente, étant donné que nombre de ces « animaux sauvages » étaient à même de provoquer des blessures graves par leurs morsures, griffures, coups de pied, ou de corne. Pour la santé et la sécurité des animaux et des hommes qui s'occupaient d'eux, il était salutaire que ces animaux soient apprivoisés, du moins jusqu'à un certain point. Tout aussi important pour le Muséum était d'éduquer les citoyens. Parmi les visiteurs, certains non seulement nourrissaient les bêtes en dépit de l'interdiction qui leur en était faite, mais aussi leur lançaient des pierres, lâchaient leurs chiens pour les poursuivre dans leurs enclos, en faisaient des objets de dérision, etc. ${ }^{14}$ Le Muséum avait également un problème persistant pour mettre bon ordre au comportement des gardiens d'animaux qui, tous, avaient dans le passé gagné leur vie en tant que montreurs de bêtes. En novembre 1795, le Muséum décida de désigner un surveillant spécial au jardin zoologique parce que les professeurs étaient trop occupés à d'autres

(13) « Nouvelles littéraires: Embellissements du Jardin des Plantes », Magasin Encyclopédique, thermidor an XI [juillet-août 1803], p. 518-520.

(14) Richard W. BURKHARDT, « La ménagerie et la vie du Muséum »... art. cit. 
tâches pour « donner à la ménagerie une surveillance attentive et journalière et que les employés de cette partie sont en quelque sorte abandonnés à eux-mêmes $»^{15}$.

J.-C. Mordant Delaunay, le bibliothécaire adjoint du Muséum fut choisi pour superviser la ménagerie. Il prit son rôle très à cœur. De ce fait, il rappela continuellement aux professeurs/administrateurs qu'il était nécessaire qu'ils accordent davantage d'attention aux besoins du jardin zoologique. Par exemple, en septembre 1796, il écrivit aux professeurs, exposant que « La Ménagerie n'est pas l'objet le plus important du Muséum, mais elle est peut-être celui qui intéresse le plus généralement toutes les classes, et à cet égard elle mérite votre attention $»^{16}$. Ce point vaut la peine d'être souligné. Puisque les images avaient de l'importance, et que la ménagerie semblait être la partie préférée du public au sein du Muséum, ce dernier devait s'assurer que les images qu'il présentait soient positives. Pourtant, dans leurs pratiques scientifiques quotidiennes, les professeurs avaient tendance à se concentrer sur des spécimens morts plutôt que vivants. D'autres individus avaient la charge de s'occuper de la vie de tous les jours du jardin zoologique, qui, à son tour, leur procurait la possibilité de tenir un rôle prépondérant dans la formulation des significations qui ressortaient de la ménagerie. L'une de ces personnes fut « le gardien des animaux féroces », Félix Cassal. Bien que ce dernier ne sût pas écrire - à part signer de son nom lettres et documents par un griffonnage d'enfant sa voix s'avère capitale pour notre récit.

\section{Les lions de la ménagerie}

Le premier lion du Muséum fut celui qu'avait vu Bernardin de SaintPierre à Versailles. Transféré de ce lieu au Muséum en avril 1794, ce lion, accompagné de son chien, attira l'attention de Georges Toscan, l'homme que le Muséum devait bientôt choisir comme premier bibliothécaire. (Oui, les deux bibliothécaires du Muséum jouèrent un rôle primordial au cours de l'histoire des débuts de la ménagerie.) Toscan se targuait d'être un ami de la nature ; il était rédacteur à la nouvelle revue républicaine, $L a$ Décade philosophique, politique, et littéraire. Comme Bernardin, Toscan s'opposait à la cruauté envers les animaux et à l'esclavage, soutenait que la contemplation des harmonies de la nature était la seule voie sûre vers le

(15) AN, AJ/15, séance du 24 brumaire an IV, procès-verbaux des assemblées des professeurs (ci-après PV), AJ/15/97, p. 92.

(16) $\mathrm{AN}, \mathrm{AJ} / 15$, séance du 14 vendémiaire an $\mathrm{V}$, minutes des procès-verbaux des assemblées des professeurs (ci-après MPV), AJ/15/580. 
progrès moral de l'homme, et croyait que l'amélioration de la manière dont les hommes traitaient la nature conduirait aussi ces derniers à se mieux comporter les uns vis-à-vis de leurs semblables ${ }^{17}$. Bien que la botanique ait été la discipline d'histoire naturelle préférée de Toscan, le couple lion - chien s'imposa à lui comme sujet incontournable car cela lui permettait de montrer que, si le lion de la ménagerie avait perdu quelque chose en captivité, il avait, dans le même temps, gagné autre chose " par l'influence de la société et des bienfaits ${ }^{18}$.

Toscan faisait sienne l'idée que tout ce dont l'homme avait eu besoin pour domestiquer les animaux était la bienveillance de son cœur, un des concepts de Buffon ${ }^{19}$; une bonne partie de sa représentation du caractère naturel du lion reflétait aussi l'influence de Buffon. L'article de Toscan chantait les louanges de la simplicité de la nature et les bienfaits de la générosité et de la société. Comme Bernardin avant lui, il rapporta comment le chien et le lion jouaient ensemble. De même, il observa la façon dont le chien avait très envie de plaire aux hommes et semblait ne pas ignorer que ces derniers aimaient le voir lui et le lion mener ensemble une existence heureuse ${ }^{20}$. L'élément nouveau dans ce que Toscan relata fut la mort du chien, quelques mois après l'arrivée du duo à Paris, et comment le lion réagit alors :

«Le Lion privé de son ami, l'appeloit sans cesse par de sombres rugissemens : bientôt il tomba dans une profonde tristesse ; tout le dégoûtoit ; ses forces et sa voix s'affoiblissoient par degrés. » Imaginant que la présence d'un autre chien de compagnie pourrait profiter au lion, le personnel en introduisit un nouveau dans la cage du lion. Ce dernier tua immédiatement le chien. On ne renouvela pas l'expérience car, comme l'expliqua Toscan : « En effet, ce n'était pas un chien qu'il regrettait : c'était un ami. Le tems qui console a calmé sa douleur, et lui a rendu la santé et les forces : mais il n'a pu effacer ses regrets $»^{21}$.

L'écrit de Toscan sur le lion et le chien fut bientôt étayé par un récit fourni par l'homme qui avait été le directeur de la compagnie d'Afrique

(17) Voir Georges ToscAN, «Sciences. Histoire naturelle. Vues générales », La Décade philosophique, littéraire et politique, 1 (10 floréal an II), p. 1-7. Ceci fut repris dans Georges TosCAN, L'Ami de la nature, ou choix d'observations sur divers objets de la nature et de l'art, Paris, Crapelet, an VIII (1800).

(18) Id., « Histoire du lion et du chien », La Décade philosophique, 3 (an III), 129-137, 193-199, citation p. 130 ; Id., L'Ami de la nature... op. cit.

(19) Ibidem., p. 137.

(20) Ibid., p. 197-198.

(21) Ibid. p. 198-199. 
au Sénégal, et dans la maison duquel le lion et le chien avaient grandi ensemble. Parmi les trésors de cet exposé on trouva mention du nom donné localement au lion («Woira »), une description de la liberté totale que l'on avait octroyée à l'animal, et une image du lion accompagné de « moutons, chiens, chats, singes, autruches, oies, canards, dindons, poules, chevaux, perruches » tous vivant en bonne entente, et au cours de froides nuits d'hiver dormant «pêle-mêle et les uns sur les autres, dans les écuries, avec les chevaux. Le Lion dormoit au milieu, et les Nègres qui me servoient alloient également se coucher parmi eux, pour chercher la chaleur $»^{22}$.

Le récit de lion et du chien était communément présenté comme une leçon sur les avantages d'un traitement bienveillant et d'un engagement social. Nous pouvons aussi utiliser ce cas pour mettre en relief les conditions de vies peu satisfaisantes dans les premiers temps de la ménagerie. Toscan attribua la mort du chien à une plaie causée par le fait qu'il se couchait le dos contre un mur humide ( on s'aperçut trop tard pour y porter remède ») ${ }^{23}$. Le lion mourut deux ans après d'une " maladie vermineuse » contractée à la suite de la consommation de viande de chevaux malades, ce qui, à une époque de grande pénurie, était tout ce que le Muséum pouvait se procurer pour nourrir ses carnivores ${ }^{24}$.

L'épisode du lion et du chien souligne en plus le manque relatif d'attention des professeurs pour la ménagerie à ses débuts, pour autant que l'on puisse le dire d'après un moment saisi dans les minutes de l'assemblée professorale du 4 brumaire an III (25 octobre 1794). À cette réunion, qui eut lieu quatre jours après la parution de la première livraison de l'article de Toscan sur le lion et le chien, Daubenton, le directeur du Muséum, proposa à ses collègues que Nicolas Maréchal, le peintre animalier de cette institution, reçoive l'autorisation de faire un tableau du lion et du chien ensemble. Daubenton voulait que le « fait remarquable » de l'amitié de ces animaux soit conservé pour l'avenir. Ses collègues professeurs acceptèrent volontiers cette proposition. Ce faisant, ils montrèrent qu'ils ne savaient pas que (1.) Maréchal avait déjà peint un tableau du duo (qui avait inspiré la gravure qui accompagnait l'article de Toscan), et que (2.) le chien était

(22) Id., « Histoire du lion et du chien ; supplément », La Décade philosophique, 5 (an III), 257-261.

(23) Id., « Histoire du lion »... art. cit., p. 198.

(24) Le numéro du 10 thermidor an IV, vol. 10 (28 juillet 1796) de La Décade philosophique donne l'information que la mort du lion venait de survenir (p. 235). L'identification par Delaunay de la cause de la mort du lion apparaît dans sa lettre du 14 ventôse an VI aux professeurs du Muséum, séance du 14 ventôse an VI, AN, MPV, AJ/15/581. Voir aussi Georges TosCAN, L'ami de la Nature... op. cit., p. 47 (note) 
déjà mort ${ }^{25}$. L'ignorance des professeurs à propos de la mort du chien laisse supposer qu'ils n'étaient pas des visiteurs assidus de la «ménagerie provisoire ».

Trois mois plus tard, les lions occuperaient à nouveau l'esprit de Daubenton, quoique dans un contexte complètement différent. Le 7 pluviôse an III (26 janvier 1795), le vénérable professeur se tenait sur le podium de l'amphithéâtre récemment achevé du Muséum pour donner une conférence aux étudiants de l'École Normale. Le sujet en était la manière simple, sans fioritures, avec laquelle l'on devrait écrire ou parler lorsque l'on présentait des faits d'histoire naturelle. Soulignant que l'on ne devrait jamais sacrifier la précision scientifique aux effets rhétoriques, Daubenton cita le cas d' " un orateur très-célèbre et très-moderne, grand philosophe et grand écrivain dans le genre sublime » qui «n'a pu faire l'histoire du lion, sans se livrer à des préjugés, qu'il énonce avec une force et un agrément qui séduisent presque tous les lecteurs $»^{26}$. Sans nommer l'auteur qu'il avait en tête (il s'agissait de Buffon, son ancien supérieur au Jardin du Roi), Daubenton exposa la manière dont cet écrivain avait dépeint le lion comme une créature noble - en fait comme le roi des bêtes. Il affirma devant ses auditeurs qu'il ne pouvait en être ainsi. Selon ses termes, « le lion n'est pas roi des animaux : il n'y a point de roi dans la nature $\gg^{27}$.

On dit que ces mots prononcés par Daubenton enflammèrent son auditoire. Dans une note de la rédaction accompagnant la transcription de la sténographie de son intervention, on lit: «Les mains, les yeux, les physionomies, les acclamations, les attitudes, rendaient à l'envi la même opinion, ou plutôt, le même sentiment imprimé d'avance dans le fond de tous les cœurs. C'était vraiment l'esprit public, qui jaillissait dans toute sa pureté et toute sa force, du foyer de l'instruction. Jamais la haine de la royauté ne s'est manifestée avec plus d'énergie, et l'amour de la République avec plus d'éclat. » Pour le lecteur qui n'aurait pas saisi, cette note se poursuivait : "Ceux qui ne veulent pas de rois, même au milieu des bêtes féroces, ne les souffriront pas au milieu d'eux-mêmes $»^{28}$.

(25) AN, PV, AJ/15/96, séance du 4 brumaire an III, p. 165. Le nom de Maréchal n'apparut pas avec la gravure qui illustra l'article de Toscan dans La Décade philosophique mais il figura dans L'Ami de la nature de Toscan où ce dernier attribua explicitement l'illustration à Maréchal (p. 36, note).

(26) Louis Jean Marie DAUBENTON, « Sur la rédaction de l'histoire naturelle », dans Séances des Ecoles normales, recueillies des sténographes, et revues par les professeurs, Nouvelle édition, Paris, L'Imprimerie du cercle-social, 1800, Vol. 1, p. 288-302, citation p. 290.

(27) Ibidem, p. 291. Sur le commentaire de Daubenton qu'il n'existe pas de rois dans la nature, voir particulièrement Jeff LOVELAND, «Daubenton's lions : from Buffon's shadow to the French Revolution », New Perspectives on the Eighteenth Century, 1, 2004, p. 29-47.

(28) Id., «Sur la rédaction, » ... art. cit., p. 291 (note). 
Daubenton se mit à dépouiller le lion des diverses caractéristiques royales et nobles que Buffon lui avait attribuées. Le lion n'était pas un roi, continuait Daubenton, parce qu'il n'était entouré ni de flatteurs ni de courtisans. Bien au contraire, les autres bêtes fuyaient sa présence. Le rugissement du lion n'était pas aussi impressionnant qu'on l'avait affirmé auparavant : « La voix du lion est plus forte, plus rauque, plus rude, que terrible : c'est un rugissement qui a le ton plaintif d'un animal hors d'haleine ou souffrant : il se fait entendre par des sons entrecoupés, dont la force diminue successivement et s'éteint ». De même, il n'y avait pas de « colère noble » ou de « courage magnanime » dans la façon dont le lion choisissait et capturait sa proie, ni aucune noblesse particulière dans sa prétendue constance en tant qu'espèce ${ }^{29}$. Daubenton conclut par ses mots à ses étudiants : « Je n'ai insisté sur ces prestiges, que pour vous mettre en garde contre les écrits des naturalistes, qui mêlent la magie de l'éloquence à la vérité simple de l'histoire naturelle ${ }^{30}$.

La même année où Daubenton détrôna le lion, son collègue Lacepède présenta la problématique du statut du lion d'une manière différente. Lacepède s'était prudemment absenté de Paris pendant les jours les plus dangereux de la Révolution. Pourtant, dès février 1795, il était de retour, prenant en charge les responsabilités de nouveau professeur de zoologie au Muséum. Lui aussi donna bientôt une conférence à l'École normale, choisissant, entre autres thèmes, l'histoire et l'avenir des jardins zoologiques. Dans cette communication, il suggéra que, en définitive, la nouvelle ménagerie ne devrait plus abriter de lion du tout ${ }^{31}$.

Lacepède soutenait que, au lieu de montrer des bêtes dangereuses, présentant ainsi une image de la nature soumise par la force et la férocité, une nation avisée devrait insister sur d'autres sortes de créatures qui promettaient d'être utiles à la fois sur un plan matériel et moral. En étudiant leurs habitudes, leurs affections, leurs besoins, et le déroulement de leur vie, les naturalistes découvriraient comment les acclimater, soigner leurs maladies, améliorer leur viande, etc. De plus, les citoyens, observant les scientifiques occupés à de telles études, apprendraient à faire la distinction entre ce qui était vraiment utile et ce qui était seulement exaltant - et, ce faisant, ils en viendraient à comprendre le bien que les hommes de science

(29) Ibidem, p. 292.

(30) Ibid., p. 292-293.

(31) Bernard Germain de LACÉPÈDE, «Lettre relative aux établissemens publics destinés à renfermer des animaux vivans, et connus sous le nom de ménageries », La Décade philosophique, 7, 1795 - 20 frimaire an IV, p. 449-462, citations p. 453-454. 
apportaient à la nation. Il serait instructif que le public voie, par exemple, « le lion si fier et si superbe, mais si inutile et si dangereux, céder sa place au bélier de Cachemire, si doux, si humble, si pacifique et si utile $»^{32}$.

Qu'il ait été enchanté de sa propre prose ou inquiet de ne pas paraître suffisamment utilitariste, Lacepède avait conduit son argumentation au-delà de ce qui était nécessaire d'un point de vue politique, si l'on peut en juger d'après le rapport sur le Muséum qu'en fit Antoine Claire Thibaudeau à la Convention Nationale en décembre 1794. Pour que les ménageries soient « utiles à l'instruction publique, » écrivit Thibaudeau, elles devaient être « construites de manière que les animaux de quelque espèce qu'ils soient [italiques de Burkhardt] jouissent de toute la liberté qui s'accorde avec la sûreté des spectateurs, afin qu'on puisse étudier leurs mœurs, leurs habitudes, leur intelligence, et jouir de leur fierté naturelle dans tout son développement. » Les bêtes ne devaient pas être détenues d'une manière qui montrait « la flétrissure de l'esclavage, » mais plutôt d'une façon qui permettait à chacun d'admirer « la force majestueuse du lion, l'agilité de la panthère, et les élans de colère ou de plaisir dans tous les animaux. » Quant aux animaux plus paisibles, «ils pourront être placés dans des parcs un peu étendus, en partie ombragés par des arbres, et tapissés de verdure propre à les nourrir $»^{33}$.

Pour le « gardien des animaux féroces » du jardin zoologique, Félix Cassal, il était évident qu'un tel établissement eût des lions. Lorsque celui qui s'y trouvait mourut en juillet 1796, personne à part lui ne ressentit plus cruellement cette perte. Ceci laissa la ménagerie presque dépourvue d' «animaux féroces». Dans un dialogue inventé, mais parfaitement plausible, proposé par Louis François Jauffret dans son guide du Jardin des Plantes pour la jeunesse, on trouve Cassal s'adressant à son jeune visiteur : "Quand le public vient la visiter, je suis comme honteux de n'avoir à lui montrer que des animaux ordinaires. Je voudrais pouvoir vous dire à présent : Voilà le tigre, voilà la panthère, voilà le léopard, voilà le chacal, voilà l'hiène, voilà le lion $»^{34}$. Pour Cassal, pourtant, il y avait plus que de

(32) Ibidem., p. 456.

(33) Antoine Claire Thibaudeau, « Rapport fait ... sur le Muséum national d'histoire naturelle, » séance du 21 frimaire an III, dans James GuILlaUME (dir.), Procès-Verbaux du Comité d'Instruction Publique de la Convention Nationale, tome 5, Paris, Imprimerie nationale, 1904, p. 275 281.

(34) Louis François JaUfFret, Voyage au Jardin des Plantes, Paris, Houel, an VI, p. 126-127. Le livre décrit des événements datant de 1796 comme s'ils étaient contemporains, mais en réalité le livre ne parut pas sous forme imprimée avant le printemps 1798. 
la fierté en jeu. Il était inquiet car, si le Muséum ne reconstituait pas sa collection d' « animaux féroces », il n'aurait plus besoin de ses services ${ }^{35}$.

Plus tard en 1796, Cassal suggéra que le Muséum l'envoie en Afrique du Nord pour qu'il aille y chercher des bêtes pour la ménagerie. Le Muséum en accepta le principe et écrivit au ministre de l'Intérieur, faisant valoir que le renouvellement de la collection d'animaux était une question d'honneur national ( «...la Ménagerie du Muséum est dans un état de dénuement peu convenable pour un établissement qui doit honorer la Nation».). Cette institution fournit une longue liste d' « animaux curieux et utiles » que l'on pouvait s'attendre à trouver dans le royaume de Tunis. Au sommet de la liste figurait le lion ${ }^{36}$.

Reconnaissant que la ménagerie dans son état d'alors était «fort incomplète », le ministre donna son accord pour favoriser l'expédition de $\mathrm{Cassal}^{37}$. Tout au long des préparatifs pour cette entreprise, grandes furent les espérances ${ }^{38}$. Malheureusement, le départ de Cassal pour Tunis fut reporté de plusieurs mois du fait des atermoiements de l'ambassadeur tunisien. Puis la peste en Afrique du Nord l'empêcha de se rendre sur le terrain assez tôt afin de chasser. S'il n'y avait pas eu les animaux que les chefs régionaux lui offrirent comme cadeaux diplomatiques pour la France (ce qui ne se produisit que grâce à l'aide des consuls français sur place), Cassal aurait dû revenir quasiment les mains vides. Un couple de lions mâle et femelle donné par le Bey de Constantine, une jeune lionne du Sahara offerte par le Prince Sulabay, une gazelle, deux chameaux, et une paire d'autruches, don du Dey de Tunis, constituèrent les principaux trophées de l'expédition de Cassal. Ce dernier possédait également une jeune lionne qu'il avait achetée à un moment où il pensait que, sans cela, il aurait pu ne pas avoir un seul lion.

Tandis que Cassal patientait au cours de la quarantaine qu'il devait subir après son retour à Toulon, ses sentiments étaient partagés entre la gêne devant la faible quantité d'animaux qu'il avait rassemblés et l'euphorie eu égard à la qualité de ceux en sa possession. Soulignant ce bon côté dans une lettre à Delaunay, il manifesta son enthousiasme pour « un superbe lion

(35) Dans une lettre du 14 pluviôse an V, Cassal remerciait les professeurs qui appuyaient sa proposition de reconstituer la ménagerie, ce par quoi « [ils] me conserveront la certitude d'être attaché au Muséum national d'histoire naturelle. » AN, MPV, AJ/15/580, classée à la séance du 24 pluviôse an V.

(36) AN, PV, AJ/15/98, séance du 14 frimaire an V, p. 33-34.

(37) Voir la lettre du ministre de l'Intérieur aux professeurs du Muséum, en date du 3 pluviôse an $\mathrm{V}$, dans $\mathrm{AJ} / 15$ séance du 4 pluviôse an $\mathrm{V}, \mathrm{AJ} / 15 / 580$.

(38) Décade philosophique, 12, 10 pluviôse an V, p. 237. 
de trois ans, [et] une lionne également du même âge, qu'il seroit possible de faire co-habiter, si vous disposiez convenablement le local où étoit dans notre ménagerie le lion qui est mort. » Pour ce qui est de la lionne du Sahara, il la qualifia d'être «la plus féroce que j'ai vue de ma vie ${ }^{39}$.

Cassal voulait se rendre immédiatement à Paris après la levée de la quarantaine qui le retenait lui et ses animaux, mais le gouvernement et le Muséum étaient d'un autre avis. Ils le dissuadèrent de partir avant Pâques afin de ne pas mettre en danger la vie de ses bêtes en les transportant au cours de l'hiver. Le gouvernement avait une raison supplémentaire de le retarder : il souhaitait qu'il attende afin de pouvoir faire la jonction avec le grand convoi chargé des trésors artistiques dont les Français s'étaient récemment emparés en Italie. Le ministre de l'Intérieur tenait à ce que les animaux d'Afrique et les œuvres d'art en provenance de la péninsule arrivent à Paris ensemble, dans une magnifique procession triomphale. C'était aussi l'opinion d'André Thouin, «professeur de culture » au Muséum, l'un des commissaires chargés de collecter les objets d'art, les outils agricoles, les animaux domestiqués, etc. en Italie ${ }^{40}$. Thouin se délectait à la perspective de «l'effet moral » que produirait le spectacle de l'arrivée à Paris des œuvres artistiques venant de là-bas ${ }^{41}$. Lorsque ses fonctions de commissaire l'amenèrent à Toulon, en février 1798, il rencontra Cassal. Il fit son rapport au Muséum où il indiqua que tous ses animaux étaient en très bonne santé et que le lion était «infiniment plus beau et plus fort que celui que la ménagerie nationale a perdu $»^{42}$.

Finalement, Cassal partit avec ses bêtes pour Avignon où il attendit le convoi italien ${ }^{43}$. Puis il voyagea avec lui par voie fluviale jusqu'à Paris. Il regagna cette ville le 27 messidor an VI (15 juillet 1798), dix-huit mois et vingt-deux jours après en être parti ${ }^{44}$. Treize jours plus tard, cinq de ses

(39) Les détails de l'expédition de Cassal proviennent du sous-dossier des lettres de Cassal au Muséum intitulé « Transport d'animaux d'Afrique », AN, AJ/15/844, « Ménagerie. » Les citations ici sont extraites de la première lettre de Cassal à Delaunay après son arrivée à Toulon, en date du 29 brumaire an VI [19 novembre 1797].

(40) Sur Thouin, voir Yvonne LetouZEY, Le Jardin des Plantes à la croisée des chemins avec André Thouin, 1747-1824, Paris, Éditions du Muséum, 1989.

(41) Voir « Lettre de C. Thouin, commissaire français en Italie, sur les objets d'arts conquis dans ce pays, » La Décade philosophique, an V (1797), t. 14, p. 212-215. Aussi dans Yvonne LETOUZEY, op. cit., p. 499-501.

(42) Le Magasin encyclopédique, $3^{\mathrm{e}}$ année, tome 6, an VI (1798), p. 117-118.

(43) Les lettres de Cassal du $1^{\text {er }}$ nivôse an VI (de Toulon) et du 20 ventôse an VI (d'Avignon) révèlent son irritation à devoir attendre. $\mathrm{AN}, \mathrm{AJ} / 15 / 844$.

(44) « Rapport de la compte [sic] de C. Cassal, » AN, MPV, AJ/15/581, séance du 14 fructidor an VI. L'exposé indique que Cassal partit de Paris le 10 pluviôse an V. Il consigne, de façon erronée, que la date de son retour dans la capitale est le 27 thermidor, au lieu du 27 messidor an VI. Cassal fournit son rapport officiel à l'administration le 4 thermidor an VI. AN, PV, AJ/15/99, p. 9. 
animaux - le couple de lions, la lionne du Sahara et les deux chameaux figurèrent en bonne place dans l' " entrée triomphale des objets de sciences et d'arts recueillis en Italie » au cours des « fêtes de la liberté ». Également dans le « cortège » se trouvait un des trois ours que les Français avaient soustraits comme trophées de guerre de la fosse aux ours de Berne. Deux dromadaires, dont on s'était emparé trois ans auparavant en Belgique, faisaient aussi partie du défilé. On distinguait clairement tous les animaux (les lions et l'ours étaient transportés dans des cages sur des chariots ; les chameaux et dromadaires avançaient sur leurs pattes). Pendant ce temps, la plupart des objets d'arts, à part les célèbres chevaux de Saint-Marc, se trouvaient dans des caisses et hors de vue. Ainsi les animaux jouèrent-ils un rôle primordial dans ce spectacle ${ }^{45}$.

En dehors des cérémonies protocolaires de la fête, « les Auteurs des dîners de Vaudeville » cherchèrent à traduire en chansons la nouvelle suprématie culturelle de la France. L'une d'entre elles avait pour refrain : « Rome n'est plus dans Rome, Elle est toute à Paris ». Les animaux de la ménagerie firent leur apparition dans le deuxième couplet d'une autre chanson :

«L'Afrique a fourni de lions

Notre Ménagerie :

Grâce à Berne nous y voyons

Les Ours de l'Helvétie.

Nous avons aussi de Saint-Marc

Les Chevaux, et j'espère

Que, bien bridé le Léopard

Nous viendra d'Angleterre $»^{46}$.

Pour le gouvernement et le public, les lions de Cassal faisaient partie de la représentation de la gloire nationale. Pour leur gardien lui-même, ces animaux lui garantissaient son poste. Grâce aux quatre lions d'Afrique du Nord et aux trois ours de Berne, la collection de bêtes féroces avait été rajeunie. Cassal porta un intérêt particulier au couple de lions. Au mâle, il donna le nom de « Marc», et de «Constantine » à la femelle.

(45) Voir Fêtes de la liberté, et entrée triomphale des objets de sciences et d'arts recueillis en Italie. Programme, Paris, Imprimerie de la République, thermidor an VI ; Patricia MAINARDI, « Assuring the Empire of the future : the 1798 Fête de la Liberté », Art Journal, 48, 1989, p. 155-163; et Richard W. BURKHARDT, « Civilizing specimens and citizens », art. cit.

(46) «Couplets chantés à la fête des arts », Décade philosophique, 18, an VI, p. 232-235. 
Tous les auteurs qui s'exprimèrent à propos de la ménagerie au tournant du siècle eurent quelque chose à écrire à propos de cette paire de lions, que les noms donnés par Cassal soient indiqués ou non (la tendance fut que l'on trouva les noms affectueux dans les écrits populaires, mais pas dans les publications des scientifiques). Deux éléments à propos des lions de Cassal firent en particulier impression sur le public. Le premier était les rapports de confiance existant entre leur gardien et eux, et combien ces animaux semblaient l'aimer. Le deuxième fut que la lionne donna naissance, en novembre 1800, à trois lionceaux en bonne santé.

Toscan donna le ton sur le premier de ces points, décrivant en 1799 comment le lion « vient de donner à son gardien, ou plutôt son ami, des marques d'affection et de reconnaissance qui méritent d'être rapportées ». Il relata une longue anecdote sur ce qu'il advint lorsque, à un moment donné, Cassal, malade pendant plusieurs jours, fut remplacé. Toscan dépeignit la tristesse du lion du fait du changement : "L'inquiétude, la souffrance intérieure de cet animal, firent croire qu'il étoit véritablement atteint de maladie ; mais personne n'osoit l'approcher ». Lorsque Cassal finit par revenir, le lion bondit à ses côtés :

« Il se dresse contre Félix, le presse de ses pattes, lui lèche les mains, le visage, et rugit de plaisir. La femelle joyeuse accourt aussi ; le lion la repousse ; il se fâche ; il craint qu'elle ne lui dérobe des faveurs dont il est jaloux. Une rixe alloit s'élever ; mais Félix entre dans la loge pour contenter l'un et l'autre : il les caresse tour-à-tour, et reçoit alternativement leurs caresses. »

À propos de Cassal et de la paire de lions Toscan poursuivait : « On le voit fréquemment au milieu de ce couple redoutable, dont il a su enchaîner la puissance. Il converse tantôt avec le mâle, tantôt avec la femelle ; il les flatte, il les baise sur la bouche. Veut-il qu'ils se séparent, et que chacun se retire dans sa loge ? Il n'a qu'à dire un mot. Désire-t-il qu'ils se couchent à la renverse, pour montrer aux assistans leurs pattes armées de griffes terribles, et leurs gueules hérissées de dents menaçantes ? Au moindre signe, ils se mettent sur le dos, tendent complaisamment leurs pattes l'une près l'autre, ouvrent la gueule; et pour récompense, obtiennent la faveur de lui lécher la main ${ }^{47}$.

Depuis le moment où il avait acquis la paire de lions en Afrique du Nord, Cassal avait eu l'idée de les accoupler. Lorsque la femelle, à 
l'âge d'environ cinq ans, vint en chaleur pour la première fois, il mit les deux animaux ensemble. Ils se comportèrent calmement d'abord, puis se produisit un combat d'une semaine dont la férocité laissa pantois au moins l'un de ceux qui l'observèrent ${ }^{48}$. La lionne s'avéra pleine, sauf qu'elle avorta deux mois plus tard, mais le seul fait qu'elle avait été gravide dans un environnement européen suscita un concert de louanges dans la presse ${ }^{49}$. Cependant, cet enthousiasme ne serait rien, comparé à la joie qui accueillit la mise bas, chez la lionne, de trois lionceaux mâles en bonne santé, quelque quatre mois plus tard, le 19 brumaire an IX (10 novembre 1800). Cassel nomma « Marengo » le premier d'entre eux, d'après le site de la victoire militaire de la France cinq mois plus tôt. Il donna aux deux autres lionceaux les noms de « Jemmapes » et « Fleurus », triomphes français aux Pays-Bas en 1792 et 1794 .

Les visiteurs vinrent en masse voir les lionceaux nouveau-nés et leur mère. Le peintre Maréchal commémora les naissances en un tableau particulier montrant la lionne et sa progéniture dans leurs quartiers, la mère observant attentivement un spectateur qui regardait le groupe avec insistance. Un dessin de Jean-Baptiste Huet représenta la famille du lion avec Cassal tenant un des lionceaux. Napoléon et Joséphine Bonaparte vinrent chacun rendre visite à la mère et ses petits. Dans ses mémoires, la duchesse d'Abrantès, qui se rendit auprès de la lionne et ses lionceaux en compagnie de Joséphine, relata que Cassal donna un petit à Madame Bonaparte pour qu'elle le porte. Pour ce qui est de Napoléon, il « caressa la lionne et causa avec Félix sur toutes ses bêtes avec une aisance aussi grande, une connaissance de tout ce qui regardait leur hygiène et leur conduite, qu'en vérité on aurait pu croire qu'il avait fait de cette partie du savoir une étude particulière $»^{50}$. La duchesse fit également une remarque à propos de Cassal, selon laquelle il était « à sa manière un homme extraordinaire. » Les histoires dont il amusait les dames étaient difficiles à croire, d'après elle, mais il «était toujours intéressant à entendre : tout ce qu'il disait ensuite n'était pas mensonge, si tout n'était pas vérité, et l'on trouvait à

(48) Voir Frédéric CUVIER, « Le lion de Barbarie, la lionne et ses petits », p. 2, dans Étienne GEOFFROY-SAINT-HILAIRE et Frédéric CUVIER, Histoire naturelle des mammifères, avec des figures originales enluminées, dessinées d'après des animaux vivants. 7 vol. Paris, C. de Lasteyrie, 1818-1842. Note : la pagination est distincte pour chaque article dans cet ouvrage.

(49) Denys MONTFORT, «Sur la lionne de la ménagerie », Magasin encyclopédique, 6, tome 2, An VIII - 1800, p. 238-239.

(50) D'ABRANTÈs, Mémoires de Madame la Duchesse d'Abrantès, ou souvenirs historiques sur Napoléon, la Révolution, le Directoire, le Consulat, l'Empire et la Restauration, tome 4', Paris, Ladvocat, 1831, citation p. 155. 
s'amuser et à s'instruire en l'écoutant $»^{51}$. La duchesse rapporta aussi que, quand Cassal, au milieu d'une de ses histoires, arrivant à ce qu'il désignait comme la partie la plus étonnante, « Napoléon lui dit en lui frappant la tête : "Félix tu mens, mon garçon, il n'y a pas de crocodiles dans l'endroit que tu désignes, et il n'y en a jamais eu. Mais c'est égal ; continue toujours ton histoire" $\gg^{52}$.

Nous soupçonnons Cassal d'avoir, à cette époque, raconté une autre histoire peu crédible qui s'ancra plus durablement dans les esprits. Elle concernait le groupe de tigres du Bengale, de léopards et autres animaux que le Muséum avait acheté à Londres en 1800. Lucien Bonaparte, ministre de l'Intérieur, réserva des fonds pour cet achat, convaincu que ces animaux procureraient à la ménagerie l'éclat qui lui était indispensable. Le vendeur était Gilbert Pidcock, le célèbre marchand d'animaux britannique. Faux était le récit qui circula à Paris à ce propos : que ces bêtes avaient autrefois appartenu au jardin zoologique de Tippoo Sahib, le célèbre sultan de Mysore. Nous croyons que l'un des gardiens d'animaux à la ménagerie, très vraisemblablement Cassal (mais peut-être Bernard Lousardi), avait concocté cette histoire pour donner du panache à son boniment de bateleur ; à partir de là, elle fut reprise dans plusieurs guides non autorisés de l'époque, pour être simplement répétée, des années plus tard, par des historiens sans méfiance ${ }^{53}$.

Il faut souligner que ce fut Cassal, et non l'un des professeurs au Muséum, qui s'occupa de l'accouplement des lions (leur gardien essaya aussi, mais sans succès, de faire se reproduire les deux tigres achetés à Londres). En 1801, les professeurs se plaignirent expressément d' « essais d'accouplemens et autres expériences faites sans ordre et sans témoins instruits » comme étant une des nombreuses façons dont les gardiens d'animaux se comportaient : agissant avec trop d'indépendance, ils outrepassaient leurs fonctions ${ }^{54}$. Mais les zoologistes menèrent bel et bien une expérience avec les lionceaux. Ils firent castrer l'un d'entre eux pour voir l'effet que cela aurait sur son comportement. Ils prévoyaient que cela atténuerait sa férocité. Contrairement aux attentes, ce petit se montra

(51) Ibidem., vol. 4, p. 151-152.

(52) Ibid., vol. 4, p.155.

(53) Relater l'intégralité de cette histoire prendrait plus de place que ce dont nous disposons ici. Nous la traiterons dans notre livre à paraître sur la ménagerie.

(54) AN, MPV, AJ/15, séance du 7 thermidor an IX ; AN, MPV, AJ/15/586 ; AN, PV, AJ/15/102, p. 34-36. 
plus difficile à gérer que ses compagnons de portée ${ }^{55}$. Cependant, les effets à long terme de l'opération ne purent être étudiés, car aucun des lionceaux ne vécut jusqu'à l'âge adulte ; tous périrent vers l'âge de un an à la suite de difficultés liées à leur dentition.

Parmi les auteurs qui écrivirent à propos de la lionne et ses lionceaux se trouva Lacepède. Il le fit dans le bel ouvrage intitulé La Ménagerie du Muséum national d'histoire naturelle, qui commença à paraître, sous forme de livraisons, en $1801^{56}$. Cette publication comportait des gravures de tableaux de Maréchal accompagnées de textes écrits par trois des professeurs du Muséum : Lacepède, Georges Cuvier, et Étienne Geoffroy Saint-Hilaire. Lacepède se réserva l'exposé d'introduction à l'ouvrage (sur l'histoire des ménageries) outre les articles sur la lionne et le tigre. Ce qui est fort intéressant, bien que son discours introductif reprenne différents thèmes de ses communications antérieures sur les jardins zoologiques, est la disparition de la suggestion qu'il avait faite dans le passé sur la manière dont les citoyens devraient s'instruire en se satisfaisant de la contemplation de moutons plutôt que de lions. Dans son article sur la lionne, de fait, il jubilait indéniablement à l'évocation du nombre de lions qu'avait eu la ménagerie. Il en recensa onze - dix en vie (un adulte mâle, quatre femelles, trois lionceaux mâles, deux lionceaux femelles) et un mort (le premier mâle venu du Sénégal et de Versailles). Lacepède supposait avec fierté que c'était le plus grand effectif de lions dans un jardin zoologique « depuis que l'on a consacré des ménageries au progrès des sciences physiques $»^{57}$. Grâce à ce grand nombre d'animaux à observer, il avait été possible, d'après lui, de découvrir, sur cette espèce, des phénomènes dont on n'avait pas eu connaissance dans le passé. La durée de gestation de la lionne de 108 jours semble avoir été le fait essentiel présent à son esprit. Ce que Lacepède ne pouvait savoir fut que, dans l'intervalle de trois ans seulement, cette ménagerie serait surchargée de lions, conséquence de cadeaux diplomatiques dont elle n'avait que faire. Déjà en 1801, elle négociait avec l'Angleterre l'échange d'une de ses lionnes contre quelques kangourous $^{58}$.

(55) Bernard Germain de LACÉPÈDE, «La lionne » p. 7. dans La Ménagerie du Muséum national d'histoire naturelle, ou description et histoire des animaux qui y vivent ou qui y ont vécu ; par les citoyens Lacépède et Cuvier, avec des figures peintes d'après nature, par le citoyen Maréchal, peintre du Muséum, Paris, Miger, 1801.

(56) Florence F. J. M. PIETERS, « "La ménagerie du Muséum national d'histoire naturelle" par Lacépède, Cuvier \& Geoffroy », Bijdragen tot de Dierkunde, 51, 1981, p. 219-249.

(57) Bernard Germain de LACÉPÈDE, «La lionne » ... art. cit., p. 1.

(58) En 1804, Frédéric Cuvier dénombra neuf lions adultes à la ménagerie : les quatre ramenés d'Afrique du Nord par Cassal, deux mâles et une femelle donnés par le Dey d'Algérie au Premier Consul 
À la lecture du texte de Lacepède sur la lionne on a l'impression que la critique de Daubenton à propos du lion de Buffon n'avait jamais été formulée. «Buffon a peint le Lion » écrivait Lacepède, «tâchons d'esquisser quelques traits de la Lionne $»^{59}$. Nous ne suivrons pas Lacepède dans ses envolées rhétoriques en émulation de son idole, mais nous noterons tout du moins qu'il dépeignit le lion comme un animal avec un « empire », de fait, comme une « espèce-roi $»^{60}$. Comme Buffon l'avait fait, Lacepède décrivit la manière dont l'homme avait contesté l'empire du lion et avait obligé l'espèce à se retirer dans des lieux désolés et inhospitaliers. Lacepède réitéra également l'histoire bien connue du lion et du chien venant de Versailles, puis fit état d'un cas plus récent à la ménagerie, celui d'une lionne qui vivait aussi avec un chien. Il le présenta comme un exemple de la façon dont des relations de bonne compagnie permettaient à des animaux en captivité de supporter la perte de leur indépendance.

Lacepède emboîta encore le pas de Buffon lorsqu'il représenta le tigre comme l'équivalent du lion en cruauté : de par sa nature profonde, il avait besoin de déchiqueter sa proie « pour chercher dans ses lambeaux encore palpitants tout le sang dont il est altéré ». Alors que les lions de la ménagerie faisaient montre parfois d'une certaine "gaieté folâtre », les tigres semblaient "presque immobiles sur leurs pieds, ou couchés comme dans une sorte de contrainte pénible et de rêverie sinistre, » ne délaissant « cet état d'anxiété et de silence sombre, que pour ressentir une joie féroce à la vue des aliments qui leur sont destinés ». Ce que Lacepède avait précédemment écrit au sujet de l'importance de ne pas présenter aux citoyens des images de force et de contrainte ne ressemblait en rien à son évocation des rapports de Cassal avec les tigres de la ménagerie. D'après Lacepède, Cassal n'était pas parvenu à rendre ces animaux un tant soit peu plus dociles mais il avait réussi « à les faire obéir à quelques-uns de ses ordres. Il les force à se coucher quand ils sont debout, et à se lever quand ils sont couchés. Mais ils ne cèdent qu'en grondant, ils n'obéissent qu'avec lenteur ; et pour leur imposer cette soumission involontaire, il faut

vers la fin de l'an XI (ils arrivèrent en décembre 1803) et deux femelles rapportés par l'expédition Baudin en 1804. Voir Notice des animaux vivans de la Ménagerie, leur origine et leur histoire dans cet Etablissement, Paris, Levrault, an XII- 1804. La paternité de Cuvier de ce volume anonyme est avérée. Il en présenta un exemplaire au Muséum lors de la séance du 13 thermidor an XII ( $1^{\mathrm{er}}$ août 1804). En 1802 les représentants du Muséum vendirent une lionne en Angleterre et revinrent avec un couple de kangourous. Cette lionne a pu être celle-là même qu'un corsaire français avait prise sur un bateau anglais et offerte au gouvernement dans les premiers mois de 1797 ; à ce propos voir La Décade philosophique, 12 (an V), p. 496 et Georges TosCAN, L'Ami de la Nature, op. cit., p. 283.

(59) Bernard Germain de LACÉPÈDE, « La lionne »..., art. cit., p. 1.

(60) Ibidem, p. 3. 
qu'il grossisse sa voix, qu'il crie avec force, qu'il les menace d'un fouet redoutable ». Lacepède fut satisfait de trouver une morale à ceci : « Ainsi, parmi les animaux, comme dans l'espèce humaine, la crainte seule fait fléchir les tyrans ${ }^{61}$.

Le style écrit de Georges Cuvier présentait un contraste notoire par rapport à celui de Lacepède. Dans son article sur le lion pour La Ménagerie du Muséum national d'histoire naturelle, il s'abstenait d'user d'expressions fantaisistes concernant le caractère du lion; il proposait plutôt une vue d'ensemble pertinente de récits sur le lion depuis l'Antiquité jusqu'à son époque, décrivant ce que l'on savait de fiable à propos de cette espèce. Il est intéressant de noter qu'il conclut par «quelques faits que nous tenons du gardien Félix Cassal ». D’après la légende, le lion avait peur du chant du coq et du grognement du porc. Pour ce qui est du coq, Cassal affirmait que « bien loin que le Lion ait peur du chant du Coq, il prend le Coq lui-même, et on lui en a vu dévorer deux ou trois en quelques minutes ». En ce qui concerne le porc, le sanglier était la principale proie du lion en Afrique du Nord et Cassal avait vu l'un d'entre eux emporter un porc sauvage « comme un loup emporterait un Mouton ». Il relatait en outre que les lionnes en Afrique du Nord mettaient bas dans des lieux marécageux où il était facile d'attraper des proies venues chercher de l'eau, et que les mâles aidaient à pourvoir les jeunes en nourriture. Ceci semblait suggérer que les lions menaient une vie monogame, conclusion étayée, d'après Cuvier, par l'exemple du lion du Muséum, « qui a refusé toutes les femelles qui lui ont été offertes, exceptée celle à laquelle il s'est attaché ${ }^{62}$.

Que Georges Cuvier cite les observations de Cassal était un témoignage de plus des qualités professionnelles du gardien des animaux féroces. Pourtant, l'avenir de Cassal à la ménagerie s'avéra être limité. Au cours des premières années du nouveau siècle, des plaintes émanant du public commencèrent à s'accumuler contre lui. Parmi elles figurait le reproche qu'il avait gardé les trois lionceaux hors de la vue des visiteurs, refusant de les leur montrer, sauf s'ils payaient pour avoir le privilège de les regarder. Il semble qu'il avait également demandé de l'argent aux spectateurs qui souhaitaient le voir entrer dans la cage des lions. Lorsque, en 1801, les professeurs du Muséum prêtèrent attention aux nombreux actes de mauvaise conduite de la part des gardiens d'animaux, ils notèrent que « Chacun d'eux se regardant presque comme propriétaire des animaux qu'il soigne,

(61) Bernard Germain de LACÉPÈDE, «Le tigre », p. 3-4, dans La Ménagerie du Muséum, op. cit.

(62) Georges CUVIER, « Le lion », dans La Ménagerie du Muséum, op. cit. 
se permet d'admettre ou de refuser les curieux selon son caprice ou son intérêt, et de soustraire les animaux à la vue du public dans les heures où il devroit en jouir. Ce public est mis à contribution d'une manière quelque fois scandaleuse, et quand il ne se montre pas assez généreux, quelquefois les murmures sont assez prononcés pour être entendus $»^{63}$.

Pourtant, ce ne fut pas l'accumulation d'abus mineurs qui conditionna le sort de Cassal. Ce fut plutôt une prétendue intrigue de sa part pour faire assassiner l'administrateur des subsistances de la ménagerie (après que cet homme avait laissé entendre que le gardien volait de la viande à cet établissement). Cassal nia avoir ourdi un complot pour tuer l'administrateur (qui sortit indemne de cette machination), mais les professeurs conclurent que l'on ne devait plus lui faire confiance, et ils le licencièrent ${ }^{64}$. Il semble ironique que dans son Voyage au Jardin des Plantes, Jauffret évoque le gardien des animaux s'exprimant par ces mots : «il est moins difficile d'apprivoiser les tigres que d'apprivoiser certains hommes $\gg^{65}$.

Tout à fait par hasard, le jour même où les professeurs prononcèrent la «destitution » de Cassal (6 nivôse an XII - 27 décembre 1803), ils nommèrent Frédéric Cuvier, frère cadet de Georges Cuvier, au poste nouvellement créé de «garde de la ménagerie ». Six mois plus tard, lors de la publication de la nouvelle version du guide sur le jardin zoologique, son nouveau directeur ne put éviter de mentionner les lions et aussi Cassal. Il fit état de la tendresse et de l'affection que le couple de lions manifestait à leur gardien. À propos du lion, il écrivait « Son maitre était parvenu à le priver et à le rendre pour lui d'une docilité extraordinaire ; mais sa douceur n'était point comme celle de tant d'autres animaux, l'habitude de vivre avec des hommes et de leur obéir : cet animal ne connaissait que la voix de son maitre, et n'était doux qu'avec lui seul ${ }^{66}$. La lionne, d'après Cuvier, « avait également pris pour le cit. Cassal une très-grande affection ${ }^{67}$.

Ces remarques correspondaient à ce que Toscan avait déjà dit à propos de l'amour que les lions portaient à leur gardien. Cependant, les commentaires de Cuvier sur plusieurs autres animaux, y compris les deux autres lionnes nord-africaines de Cassal, font apparaître le gardien sous un jour différent. Concernant la lionne « du desert de la Zaara », Cuvier

(63) Jussieu, Rapport sur l'organisation de la ménagerie, AN, MPV, AJ/15/586, séance du 7 thermidor an IX.

(64) Les détails de cette affaire se trouvent dans AN, MPV, AJ/15/591. Nous la décrivons avec plus de précisions dans notre livre sur l'histoire de la ménagerie (en préparation).

(65) Louis François JAUFFRET, op. cit., p. 127.

(66) Frédéric CUVIER, Notice ... op. cit., p. 10.

(67) Ibidem, p. 14. 
écrivait : « Lorsque le cit. Cassal était chargé du soin des animaux féroces de cette Ménagerie, cette Lionne paraissait être du naturel le plus intraitable ; au seul aspect de son maître, elle bondissait dans sa loge, et menaçait en grinçant les dents de la manière la plus effrayante ». Comme Cuvier l'expliquait ensuite, toutefois «Elle montre beaucoup moins de férocité aujourd'hui envers ses nouveaux gardiens [... ]» Parler à cette lionne « avec douceur» aplanissait les difficultés. Cuvier en concluait « que sa férocité ne lui était point naturelle, et qu'elle serait peut-être très-familière aujourd'hui, si elle eût éprouvé des traitemens plus doux $\gg^{68}$.

Cuvier fut plus explicite en ce qui concerne l'autre lionne :

« si [cet animal] parait être sauvage, cela vient des mauvais traitemens qu'il a reçus : ses premiers gardiens [Cassal et son neveu] se faisant une sorte de gloire d'avoir des animaux très-féroces et intraitables en apparence sous leur garde, avaient le plus grand soin, par leurs mauvais traitemens, d'entretenir le caractère défiant et indépendant, si naturel d'ailleurs à ces sortes d'animaux $»^{69}$.

Cuvier dit la même chose de «la hyène d'Orient». La férocité excessive qu'elle manifesta au cours de ses premières années à la ménagerie était liée à la brutalité avec laquelle Cassal l'avait traitée ${ }^{70}$. Et pour le seul tigre en provenance de Londres encore en vie lorsque Cuvier rédigea sa notice, son comportement ne ressemblait en rien à ce que Lacepède avait exprimé à propos des tigres peu d'années auparavant. Selon les termes de Cuvier : "Cet animal est d'une douceur extraordinaire avec les personnes qu'il voit habituellement ; il vient au-devant de leurs caresses, en faisant entendre un bruit sourd, qu'il témoigne ordinairement lorsqu'il est joyeux $\gg^{71}$.

Il semblerait que le cas de Cassal apportait une démonstration double à ce sur quoi de nombreux auteurs de l'époque avaient insisté : qu'un comportement bienveillant entraînait la docilité chez un animal, alors qu'une attitude brutale provoquait la férocité. Pourtant personne alors

(68) Ibid., p. 17.

(69) Ibid., p. 19.

(70) Ibid., p. 27.

(71) Ibid., p. 21. Toutefois, il se peut que les tigres ne soient pas un exemple de ces animaux qui ne devinrent plus dociles qu'après le départ de Cassal. En contradiction avec l'article de Lacepède la même année, Miger, dans un écrit de 1801, précisa à propos des tigres : «Le citoyen Cassal est parvenu, par ses soins et sa manière de les traiter, à les familiariser jusqu'à les flatter avec la main. » Notice des animaux vivants, actuellement à la ménagerie du Muséum national d'histoire naturelle, Paris, 1801, p. 10. 
n'attira l'attention sur la situation de Cassal lui-même, peut-être parce que les autorités responsables du Muséum voulaient oublier ce personnage mais sans doute aussi du fait que certains exemples se révélaient ambigus ou déconcertants. C'est l'impression que paraîtrait donner le cas de Marc, ce lion qui avait eu tant d'affection pour son gardien. Cuvier ne relata pas cette histoire avant 1819. Cassal, semble-t-il, avait voulu que le lion obéisse à sa voix. Lorsqu'il ne lui fut pas possible d'obtenir la soumission de l'animal par un traitement empreint de bonté, il eut recours à la force. Cuvier écrivit :

« Ce lion avait fini par s'apprivoiser et par être très-docile pour son maître ; mais cette soumission n'avait été obtenue que par la violence. Dans sa jeunesse, il était porté à la férocité, et les bons traitements l'adoucissaient sans le soumettre ; cependant son gardien voulait qu'il obéît à sa voix, et la douceur ne réussissant pas, il eut recours aux châtiments. Ses premiers efforts furent pénibles : il fut plusieurs fois sur le point de renoncer à son projet ; mais enfin il parvint à rompre le caractère de son Lion, à lui faire connaître la crainte par la douleur, et à s'en rendre tout à fait le maître. Dès lors cet animal apprit à obéir à certains commandements, et put être offert en spectacle par son maître, que le public se plut à récompenser ; mais ce Lion porta toute sa vie les traces des coups qu'il avait reçus, les cicatrices des plaies que les coups de fouet lui avaient faites $»^{72}$.

Ce récit a encore une autre dimension qui mérite notre attention, et qui n'a pas été mise en lumière dans le passé. Peu après le congédiement de Cassal par le Muséum et la nomination de Cuvier aux fonctions de " garde de la ménagerie », ce dernier entreprit de tenir un carnet consacré à des observations sur les animaux du jardin zoologique. Après avoir inscrit le titre « le lion nommé Marc », il poursuivait en écrivant : «An 12, Nivôse » et « languissant $»^{73}$. Dans un rapport plus officiel de la ménagerie du 9 pluviôse an XII, il relata : «Le grand Lion a refusé de manger, \& il a vomi de nouveau ». Il interprétait la maladie du lion comme le symptôme du problème général auquel le jardin zoologique était confronté en tentant de garder des animaux exotiques sous un climat hostile : " Il est impossible d'attribuer d'autres causes au mauvais état des animaux carnassiers, que la saison, \& la manière dont ils sont logés. On ne pourra jamais conserver des

1819.

(72) Frédéric CuVIER, «Le lion de Barbarie » ... art. cit., p. 2. L'article est daté d'octobre

(73) Id., « Observations particulières sur les animaux de la ménagerie, an XII [1804]-1822 », Ménagerie MS, \# 16, Muséum d'histoire naturelle. 
animaux du Midi tant qu'on ne les mettra pas à l'abri de l'intempérie de nos climats $\gg^{74}$. Six mois plus tard, dans son guide sur la ménagerie, Cuvier notait que le lion n'était toujours pas à l'aise face au personnel différent autour de lui : « Ses nouveaux gardiens, malgré les soins qu'ils prennent de lui, quoiqu'il reçoive d'eux sa nourriture, et les voye à chaque instant de la journée, n'ont point encore pu lui inspirer de confiance ${ }^{75}$. Un mois et demi plus tard, le lion était mort. Le procès-verbal de l'assemblée ordinaire des professeurs du 25 fructidor an XII fit mention de ce que « Le beau lyon de la Ménagerie, nommé Marc est mort dimanche dernier entre 6 et 7 heures du matin ». Le rapport de Dufresne du laboratoire de zoologie indiqua que l'animal était « mort par les suites d'une longue maladie».

Cinq ans auparavant, Toscan avait exposé comment une brève absence de Cassal avait entraîné une souffrance chez le lion, au point que cela avait pu faire penser «qu'il étoit véritablement atteint de maladie». Encore plus loin dans le passé, Toscan avait fait état de l'affliction du premier lion de la ménagerie après la mort de son chien de compagnie. Nous n'avons trouvé aucun document de qui que ce soit en 1804 (ou plus tard) laissant entendre que l'affaiblissement et la mort du lion auraient pu être liés à la perte de son maître. Peut-être qu'une prise de conscience approfondie du côté brutal de la personnalité de Cassal - ainsi qu'il l'a manifesté dans sa manière de s'occuper de certains animaux et de conspirer pour faire assassiner un membre du personnel - aurait amoindri l'attrait d'un récit dans lequel Marc le lion dépérissait à cause du chagrin qu'il ressentait après la perte de Cassal, son maître bien aimé. Une histoire de ce genre aurait brouillé la vision habituelle qu'un traitement bienveillant suscite un comportement docile, alors que la dureté entraîne la férocité.

Les questions que se posa Cuvier, à la suite de la mort du lion, eurent une autre orientation. Dans ses carnets, il fit état de lésions découvertes sur le cadavre de l'animal. Il y avait des tubercules dans ses poumons, son foie et son côlon. Encore plus frappant était le fait que « les testicules surtout étoient dans un état d'inflammation qui les avoient presque détruits ». Cuvier s'interrogea sur la cause de tout ceci : « Il [le lion] avoit souvent couvert sa femelle, \& il en étoit séparé depuis quelque tems. Cette circonstance a-t-elle contribué à sa mort ? Est-il dangereux de développer

(74) Id., « Mouvement de la ménagerie du 9 pluviôse an XII » présenté à la séance du 11 pluviôse an XII, AN, MPV, AJ/15/591. Date $=1^{\text {er }}$ février 1804 .

(75) Id., Notice... op. cit., p. 11. 
chez ces animaux des besoins qu'on ne peut les mettre dans le cas de satisfaire toutes les fois qu'ils en éprouvent la nécessité $»^{76}$ ?

Tout au long de sa carrière en tant que " garde de la ménagerie », Cuvier allait faire montre de son souci au sujet de l'influence que les conditions de captivité des animaux de la ménagerie avaient sur leur bien-être mental aussi bien que physique ${ }^{77}$. Il exprima, par exemple, sa compassion pour la lionne après la naissance de ses trois petits, alors que « la gêne où elle était, les bornes étroites de sa loge, le mouvement des personnes qui se trouvaient sans cesse autour de sa loge » l'amenaient instinctivement à chercher à protéger ses lionceaux du danger : « elle les prenait dans sa gueule et les transportait ainsi des heures entières, en tournant dans sa loge et en manifestant une vive agitation ${ }^{78}$. De même, lorsque deux loups mangèrent leur propre portée de louveteaux, Félix Cassal interpréta cet acte comme une punition mais Cuvier eut une explication différente :

«Il est de fait qu'on n'en trouve guère d'exemples que parmi les animaux réduits en esclavage. Il est de fait encore que ces exemples ne sont pas rares et qu'ils se rencontrent même chez des animaux qui ne sont point portés, par leur naturel, à des actions féroces, comme chez les Lapins, les Cochons d'Inde, etc., etc. $»^{79}$.

Nous avons entendu des voix distinctes exprimant un avis sur le sens à donner à la présentation d'animaux dans une ménagerie. En outre, dans le cas de Cassal, nous avons vu que la question de la voix avait une signification particulière. Lorsqu'il pénétrait dans les cages des lions, il voulait tout spécialement que sa voix soit obéie. Un modeste guide de qualité médiocre qui fut de nombreuses fois publié au cours de ces années reprenait cette scène. Sa version de 1803 informait les visiteurs ainsi :

« À l'extrémité du jardin, sur les bords de la Seine, sont les animaux féroces vivans, confiés aux soins du citoyen Félix Cassal, dont la familiarité

(76) Id., « Observations particulières »... op. cit.

(77) Sur la carrière de Cuvier, voir Richard W. BuRKHARDT, Jr., « Frédéric Cuvier and the study of animal behavior », Bulletin d'histoire et d'épistémologie des sciences de la vie, 8, 2001, p. $75-98$.

(78) Frédéric CuvIER, « Le lion de Barbarie »... art. cit. p. 3.

(79) Georges Toscan, « Ménagerie », Décade philosophique, 22, 4 trim, De l'an VII, p. 323 ; Frédéric CUVIER, Notice, p. 29. 
avec eux est vraiment effrayante. À sa voix, le léopard rampe, et le roi des animaux, le Lion, semble oublier son instinct vorace $»^{80}$.

Nous pouvons admettre comme un écho persistant de la période où Cassal était en fonction au Muséum que, des années après son départ (et comme une indication de la paresse des rédacteurs du guide), les mêmes expressions aient été reprises dans ses éditions successives, dans lesquelles, toutefois, à la place du nom de Cassal, figuraient ceux des gardiens postérieurs ${ }^{81}$.

En abordant le sujet des animaux de la ménagerie au cours de la Révolution et par la suite, nous avons d'abord mis l'accent sur les lions qui s'y trouvèrent et sur la diversité des acteurs humains dont les projets individuels influencèrent la façon dont ils comprirent ces animaux. Nous avons vu la question de l'amélioration de la nature animale grâce à la bienveillance liée à l'espoir que des images positives conduiraient au perfectionnement moral des citoyens. L'épisode du lion et du chien fut, à cet égard, utile à la fois à Bernardin de Saint-Pierre et à Toscan mais il servit aussi ici à illustrer les conditions déplorables dans les premiers temps de la ménagerie et le manque d'attention relatif de la part des professeurs quant à la gestion quotidienne de cet établissement. La critique de Daubenton concernant le portrait du lion de Buffon fut un prétexte pour dire aux étudiants de l'École normale, à la fois, qu'il n'y avait pas de rois dans la nature et que les écrits sur l'histoire naturelle devaient être clairs et simples. Lacepède suggéra de ne plus exposer de lion aux regards des curieux, susceptibles qu'ils étaient de trouver, dans le spectacle d'une bête aussi féroce, un message moral erroné ; il recommanda que l'on propose plutôt aux visiteurs de voir des scènes de savants occupés à l'amélioration de la race ovine.

À la suite de la mort du lion du Muséum et du dépeuplement général de la ménagerie en « animaux féroces », Félix Cassal, le « gardien des animaux féroces », plaça les lions au sommet de la liste des espèces qu'il souhaitait recueillir en Afrique du Nord. Trois de ses nouveaux pensionnaires jouèrent un rôle incontestable au cours de la parade de la fête de la liberté en 1798 qui marqua l'arrivée à Paris des chefs-d'œuvre

(80) Le guide au Jardin des Plantes, ou description de tout ce que les Galleries, Serres, etc. du Muséum d'histoire naturelle de Paris, renferment de plus curieux, Paris, Glisau, an XI [1803], p. 25.

(81) La version de 1812 du guide comporta le nom de «Bernard Lazardi » [Lousardi], gardien ; celles de 1819 et 1823 indiquèrent Rousseau et Richard. 
artistiques d'Italie. Pour Cassal lui-même, les lions constituaient une garantie qu'il continuerait à avoir un poste au Muséum à s'occuper des animaux, à avoir la maîtrise sur leur comportement, et, ce faisant, à étonner le public.

La naissance ultérieure des lionceaux à la ménagerie attira des foules, auxquelles se joignirent le Premier Consul et Madame Bonaparte, et fut saluée comme un triomphe de la civilisation. Alors que Lacepède persistait à écrire des discours éloquents dans le style de Buffon, la gestion au jour le jour à la ménagerie suivait aussi son cours avec ses caractéristiques propres, faites de davantage de diversité, de pragmatisme et de réalisme. Dans la vie du jardin zoologique on assistait couramment à l'alternance de scènes de tendresse et de férocité, tout comme à des spectacles de liberté pour les « animaux paisibles » et de contrainte pour les « animaux féroces ». Malgré la description de Toscan du grand amour entre Cassal et ses lions, le traitement auquel le gardien soumettait ses animaux se révèle avoir eu un côté plus sinistre. Son utilisation de la violence pour obtenir la soumission du lion ne fut rapportée que des années plus tard, peut-être parce que cela allait à l'encontre de la représentation que l'on favorisait selon laquelle un traitement plein de bonté suscitait des tempéraments plus souples. Nous avons émis l'idée que son aversion pour le recours à la force de la part de Cassal aurait rendu Frédéric Cuvier peu disposé à interpréter la maladie ultérieure du lion et sa fin comme une conséquence de son chagrin à la suite du départ de son maître, même si des lésions sur le cadavre de l'animal n'apportèrent pas d'autres explications à sa mort.

Dès 1801, Lacepède avait renoncé ses projets antérieurs de mettre des moutons à la place des lions et se réjouissait du nombre de ces derniers au sein de la ménagerie. L'ironie du sort voulut que, comme des lions toujours plus nombreux arrivaient en France sous la forme de cadeaux diplomatiques, le Muséum se trouva surchargé d'animaux qui mettaient à mal ses ressources limitées, ce qui allait à l'encontre d'autres priorités. Et il y eut d'autres changements entre 1795 et le début des années 1800. En dépit de la déconstruction par Daubenton du rôle du lion en tant que « roi des animaux », dès le début des années 1800 , « le roi » était de retour, du moins dans les écrits populaires, bien que les quartiers des lions à la ménagerie du Muséum d'histoire naturelle soient restés dans un état loin d'être royal. Ainsi que l'écrivit le rédacteur du guide Jean Baptiste Pujoulx à propos de « ce bâtiment mesquin » dans lequel logeaient les « animaux féroces » : «Quoi ! c'est là, s'écrie-t-on, qu'habitent les plus puissans des animaux ? C'est là que le tigre, qui règne en tyran dans les déserts, et le lion, aussi terrible, mais plus généreux, reçoivent leurs visites » ? Pujoulx 
se hâtait d'assurer ses lecteurs que « ce logement, si peu digne du roi des animaux, de son épouse et de ses amantes » n'avait jamais été destiné à être autre chose que provisoire, « car mieux vaudrait n'avoir pas de ménagerie que de la laisser long-temps encore dans un lieu qui rappelle les loges que l'on construit à la hâte dans les foires où l'on fait voir des animaux $»^{82}$.

Lorsque le lion Marc mourut en 1804, ce fut dans ce «bâtiment mesquin », comme l'avait auparavant fait le lion nommé Woira. Dufresne, directeur du laboratoire de zoologie et taxidermiste en chef du Muséum, supervisa l'étape suivante de la transformation de Marc en spécimen zoologique. Prenant la peau du lion et les autres éléments dont il avait besoin pour obtenir une réplique aussi vraie que nature de l'animal, il prépara le tout pour être exposé en permanence dans les galeries de zoologie ${ }^{83}$. Le lion naturalisé, accompagné d'un impressionnant portrait de l'animal peint par Maréchal, allait servir de témoignage durable de l'élégance des traits distinctifs de cette bête. Quinze ans après la mort de Marc, Frédéric Cuvier désignerait ce lion comme étant « le plus bel individu de son espèce qu'eût jamais possédé notre ménagerie ${ }^{84}$. Constantine, la lionne qui avait été la compagne de Marc, était, à cette époque, toujours en vie - et toujours dans le même bâtiment. Elle resta docile et bien portante, quoique la cataracte l'ait rendue presque complètement aveugle. ${ }^{85}$ Elle vécut assez longtemps pour devenir la doyenne de la ménagerie.

Richard W. BURKHARDT, JR. Department of History University of Illinois at Urbana-Champaign 306 West Vermont, Urbana IL 61801 USA burkhard@illinois.edu Traduction de l'article assurée par Lucie Perrier

(82) Jean-Baptiste PujoulX, promenades au Jardin des Plantes, à la ménagerie et dans les galeries du Muséum d'Histoire naturelle, Paris, Guilleminet, 1803, tome 1, p. 76-77.

(83) DUFRESNE, rapport du laboratoire de zoologie, séance du 25 fructidor an XII, AN, MPV, 592. Voir aussi AN, PV, AJ/15/106, séance du 4 vendémiaire an XIII, p. 85.

(84) Frédéric CUVIER, « Le lion de Barbarie »... art. cit. p. 1.

(85) Ibidem, p. 4. 